\title{
Linear genetic programming for time-series modelling of daily flow rate
}

\author{
Aytac Guven \\ Civil Engineering Department, Gaziantep University, 27310 Gaziantep, Turkey. \\ e-mail: aguven@gantep.edu.tr
}

In this study linear genetic programming (LGP), which is a variant of Genetic Programming, and two versions of Neural Networks (NNs) are used in predicting time-series of daily flow rates at a station on Schuylkill River at Berne, PA, USA. Daily flow rate at present is being predicted based on different time-series scenarios. For this purpose, various LGP and NN models are calibrated with training sets and validated by testing sets. Additionally, the robustness of the proposed LGP and NN models are evaluated by application data, which are used neither in training nor at testing stage. The results showed that both techniques predicted the flow rate data in quite good agreement with the observed ones, and the predictions of LGP and NN are challenging. The performance of LGP, which was moderately better than NN, is very promising and hence supports the use of LGP in predicting of river flow data.

\section{Introduction}

The prediction of discharge in rivers plays an important role in river hydrology and water resources management. There are several crucial points for understanding the key statistical characteristics of available discharge data, some of which are: hydro-geological risk prevention, efficient operations of storage reservoirs for hydro-electric or other purposes, and design of many hydrogeological works (Bordignon and Lisi 2000). It is generally accepted that river flow processes, especially daily discharges, are seasonal and nonlinear, since the processes generally pronounce seasonal means, variances, and the underlying mechanisms of streamflow generation are likely to be quite different during low, medium, and high flow periods, especially when extreme events occur (Wang et al 2006).

Several linear and nonlinear methods have been applied in the prediction of discharge in rivers and successful results have been reported. These studies have focused on the prediction of discharge based on stage-discharge, rainfall-discharge or time-series of discharge relationships, using either conventional methods (Wang et al 2004; Habib and Meselhe 2006; Baiamonte and Ferro 2007; Jain 2008) or new so-called 'soft computing techniques' such as Neural Networks (NNs), Genetic Algorithms (GAs), Genetic Programming (GP), Fuzzy Logic (FL), and Machine Learning (MA) (Maier and Dandy 2000; Kisi 2004; Lopes and Weinert 2004; Cigizoglu and Kisi 2005; Kisi and Cigizoglu 2007; Aytek and Alp 2008; Guven and Gunal 2008; Guven et al 2008; Tayfur and Moramarco 2008).

For the last ten years, GAs and GP have been pronounced as alternative and robust methods in the prediction of water engineering data (Drecourt 1999; Whigham and Crapper 2001; Muttil and Liong 2004; Kalra and Deo 2007; Charhate et al 2007, 2008; Singh et al 2007; Aytek et al 2008; Deo et al 2008; Gaur and Deo 2008; Guven et al 2008; Jain and Deo 2008; Kalra et al 2008; Ustoorikar and Deo 2008). Linear genetic programmingc (LGP), which is an extension of GP is under consideration in this study. Although a few studies on GP application on hydrologic data exist in the

Keywords. Genetic programming; neural networks; daily flows; flow forecasting. 
literature, no studies have been found about the application of LGP in hydrologic data. In this sense, the present study can be considered as a pioneering study presenting the usage of LGP in the prediction of hydrologic data.

Referring to the embedding theorem of Takens (1981), the time-series modelling of daily discharge can be formulated as:

$$
\begin{aligned}
Q(t)= & f\{Q(t-n), Q(t-2 \cdot n), \ldots, \\
& Q(t-(d-1) \cdot n)\}
\end{aligned}
$$

where $n$ is the constant time delay between samples, $d$ is the embedded dimension and $f$ is a function. The objective is to find an optimal analytical model that can explain the behaviour of dynamical river flow systems. There are several conventional statistical methods to cope with this type of problem, most of them being based on ARMA-derived (Auto-Regressive Moving Average) methods. Alternatively, several heuristic models have been proposed (Bordignon and Lisi 2000; Cigizoglu and Kisi 2000; Kisi 2004; Lopes and Weinert 2004; Tayfur et al 2007; Firat 2008; Tayfur and Moramarco 2008).

Evolutionary computational methods have been successfully applied in time-series modelling of flow discharge problems, with limited number of examples. Namely, Lopes and Weinert (2004) used Gene-Expression Programming in modelling monthly time series of unregulated Rio Grande river flow at Furnas Dam in Brazil; Tayfur and Moramarco (2008) predicted hourly-based flow discharge hydrographs from level data by using GAs; Preis and Otsfeld (2008) presented a coupled model tree-genetic algorithm scheme for predicting flow and water quality constituents in watersheds.

This study proposes to employ an emerging strong evolutionary computational technique, LGP in predicting daily time series of river flow data. Also, multilayer perceptron NNs empowered by GA, and generalized regression NNs were proposed for time-series modelling of the same discharge data. Different heuristic scenarios were developed and accordingly, LGP and NN models were developed based on these scenarios. The performance of each model was compared based on the well-known statistical performance measures. The results were tabulated and illustrated in scatter and time-series diagrams.

\section{Linear genetic programming (LGP)}

GP technique is an automatic, computerized creation of computer programs in order to solve a selected problem using Darwinian natural selection. LGP, a linear variant of GP, uses a specific linear representation of computer programs. The name 'linear' refers to the structure of the (imperative) program representation, and does not stand for functional genetic programs that are restricted to a linear list of nodes only. On the contrary, genetic programs normally represent highly nonlinear solutions in this meaning (Brameier 2004). The main characteristic of LGP in comparison to conventional tree-based GP is that the expressions of a functional programming language (like LISP) are substituted by programs of an imperative language (like $\mathrm{C}$ or $\mathrm{C}++$ ).

The main characteristics of LGP are the graphbased data flow that results from a multiple usage of indexed variables (registers, $r[i]$ ) and evolving programs in a low-level language, in which the solutions are directly manipulated as binary machine codes and executed without using an interpreter (Banzhaf et al 1998; Bramier 2004). In this way the computer program can be evolved very quickly (Bhattacharya et al 2001; Brameier and Banzhaf 2001; Foster 2001).

Each individual program in LGP is represented by a variable-length sequence of simple $\mathrm{C}$ language instructions. These instructions operate on one or more registers $(r[i])$ or constants $(c)$ from predefined sets (Bramier 2003; Oltean and Groşan 2003). An example of LGP program can be:

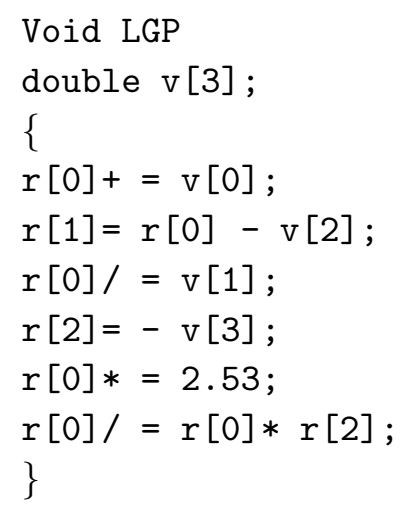

where $v[i]$ represents the input and output variables used in LGP modelling.

The function set of the system can be composed of arithmetic operations $(+,-, /, *)$, conditional branches (if $v[i] \leq v[k]$ ), and function calls $\left(f \in\left\{e^{x}, x\right.\right.$, sin, cos, tan, log, sqrt, ln, power $\}$. Each function implicitly includes an assignment to a variable $v[i]$, which facilitates the use of multiple program outputs in LGP, whereas in tree-based GP, the side effects need to be incorporated explicitly (Brameier and Banzhaf 2001). After several trials, the functional set and operational parameters given in table 1 have been used in LGP modelling during this study. 
Table 1. Parameters of the LGP model.

\begin{tabular}{lll}
\hline Parameter & Description of parameter & Setting of parameter \\
\hline$p_{1}$ & Function set & $+,-, *, /, \sqrt{ }$, power \\
$p_{2}$ & Population size & 250 \\
$p_{3}$ & Mutation frequency $\%$ & 95 \\
$p_{4}$ & Cross-over frequency $\%$ & 50 \\
$p_{5}$ & Number of replication & 10 \\
$p_{6}$ & Block mutation rate $\%$ & 30 \\
$p_{7}$ & Instruction mutation rate $\%$ & 30 \\
$p_{8}$ & Instruction data mutation rate $\%$ & 40 \\
$p_{9}$ & Homologous cross-over $\%$ & 95 \\
$p_{10}$ & Program size & Initial 80, maximum 256 \\
\hline
\end{tabular}

LGP utilizes two-point string cross-over. A segment of random position and random length is selected in both parents and exchanged between them. If one of the resulting children would exceed the maximum length, cross-over is abandoned and restarted by exchanging equalized segments (Brameier and Banzhaf 2001).

An operand or operator of an instruction is changed by mutation into another symbol over the same set. LGP also employs a special kind of mutation (called macro mutation) which deletes or inserts an entire instruction.

The fitness of an LGP individual may be computed by using the equation:

$$
f=\sum_{j=1}^{N}\left|O_{j}-E_{j}\right|,
$$

where $O_{j}$ is the value returned by a chromosome for the fitness case $j$, and $E_{j}$ is the expected value for the fitness case $j$.

In LGP, the maximum size of the program is usually restricted to avoid over-growing programs without bound (Brameier and Banzhaf 2001). In this study, the maximum size of each program has been set to 256 , starting with 80 instructions per program. This configuration has been tested for each LGP model and has been experienced to be sufficient.

The best individual (program) of a trained LGP can be converted into a functional representation by successive replacements of $v[i]$ starting with the last effective instruction (Oltean and Groşan 2003). Further details on LGP can be found in Brameier and Banzhaf (2001) and Bramier (2003).

\section{Neural networks}

In this study, the Multilayer Perceptron Neural Networks (MLPNNs) (Rumelhart 1986) with one single hidden layer, and Generalized Regression Neural Networks (GRNNs) (Specht 1991) were employed. In the architecture, logistic transfer function $\left(y=1 /\left(1+e^{-x}\right)\right)$ is utilized. The Levenberg-Marquardt back-propagation algorithm was employed to optimize the weights in neural networks. The interested reader should refer to Cigizoglu and Alp (2006) and Tayfur (2002) for further details on NN modelling.

One of the main issues in MLPNNs modelling is to obtain the optimal network architecture (number of input-number of hidden neurons-number of output) (Guven et al 2006; Guven and Gunal 2008). Most of the studies in the literature searched for optimal MLPNNs architecture by increasing the number of neurons in each trial and monitoring the performance of the model based on an error criterion. However, this method usually leads to local optimum and the resultant NN model may suffer from over- or under-predictions in validation datasets. However, GRNNs do not require an iterative training procedure as in MLPNN algorithm. GRNN approximates any arbitrary function between input and output vectors, drawing the function estimate directly from the training data (Cigizoglu and Alp 2006).

To overcome the above-mentioned issue, a genetic algorithm was used to obtain the global optimal architecture of the proposed MLPNN models. The algorithm of genetic search for an optimal NNs architecture applies a systematic evolutionary search to determine the best number of hidden neurons to get the best generalization capacity. A combination of genetic operators of selection, cross-over and mutation is used. The neural network, produced from each generation, is trained to optimize the number of hidden neurons to minimize the fitness function (error) between the observed and predicted output. The architecture is ranked and the best architecture giving the minimal error is chosen (Guven and Gunal 2008). 


\section{Auto-regressive model}

Auto-regressive (AR) model is the most widely used traditional time-series analysis. The model is usually referred to as the $\mathrm{AR}(\mathrm{p})$ model where $\mathrm{p}$ is the order model. The $\mathrm{AR}(\mathrm{p})$ model for timeseries of daily flow rate can be represented as:

$$
Q(t)=c+\sum_{i=1}^{p} \alpha_{i} Q(t-i)+\varepsilon(t)
$$

where $\alpha_{i}$ is the model parameter, $c$ is a constant, and $\varepsilon(t)$ is random error.

In this study, the $\mathrm{AR}(\mathrm{p})$ models are developed using an Excel add-in software. The model parameters are selected using Akaike's Information Criterion (AIC), given in equation (12).

\section{Study area and data used}

The dataset used in this study was obtained from the U.S. Geological Survey (USGS). The time series of daily discharge data from station 01470500 (lat. $40^{\prime} 31^{\prime} 21^{\prime \prime}$, long. $75^{\prime} 59^{\prime} 55^{\prime \prime}$ ) Schuylkill River at Berne, PA, USA are used. The drainage area at this site is $919.45 \mathrm{~km}^{2}$. Information on the daily time series for the station can be acquired from the USGS web server (http://www.usgs.gov). The data from October 1, 2002 to September 30, 2006 were chosen for training of the proposed LGP and NN models, and data of October 1, 2006 to September 30, 2007 were chosen for testing the models. Additionally, a dataset from October 1, 2007 to September 30, 2008 was reserved for application. During the analysis of data, a scattered relationship between time-lagged discharge values was observed. The discharge values ranges between 2.123 and $971.03 \mathrm{~m}^{3} / \mathrm{s}$.

\section{Development and evaluation of the models}

As explained in the preceding section, the daily discharge data between 10.01.2002 and 09.30.2006 were used as a training set (1824 sets) and the data between 10.01.2006 and 09.30.2007 as a testing set (730 sets) for calibration and validation of both LGP and NN models. Referring to Tayfur and Guldal (2006), firstly the cross-correlation between the discharge at present time $(Q(t))$ and timelagged discharges $(Q(t-1), \ldots, Q(t-d))$ was evaluated. Figure 1 shows the cross-correlation values between $Q(t)$ and each time-lagged $Q$ values. It is clearly seen from this figure that, $R$ values are

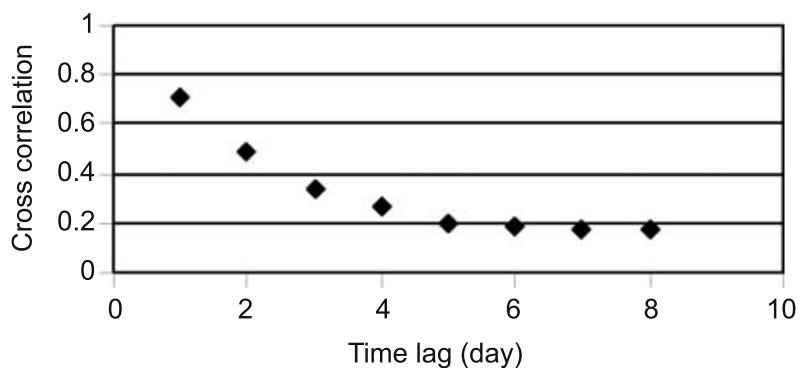

Figure 1. Cross correlations between time-lagged $Q\left(\mathrm{~m}^{3} / \mathrm{s}\right)$ values.

higher than 0.3 for four time lags $(t-1, t-2, t-3$ and $t-4$ ), and equal or lower than 0.2 after a time lag of 5 days. Similar findings were observed in LGP and NN modelling, namely, adding $Q(t-5)$ and the further time-series to the input set did not make any change in the training performance of both LGP and NN models. Therefore, it was decided to use the time series from $t-1$ to $t-4$ as input set in present modelling applications.

The relationship in equation (1) was used while developing different time-series scenarios for model development. The first scenario was $Q(t)=f(Q(t-1))$ and for each next scenario, $Q(t-2)$ to $Q(t-d)$ were added to the input set, one after another:

$$
\begin{aligned}
& Q(t)=f(Q(t-1)) \\
& Q(t)=f(Q(t-1), Q(t-2)) \\
& Q(t)=f(Q(t-1), Q(t-2), Q(t-3)) \\
& Q(t)=f(Q(t-1), Q(t-2), Q(t-3), Q(t-4)) .
\end{aligned}
$$

The testing results of LGP and NN models, in terms of coefficient of determination $R^{2}$, mean square error (MSE) and mean absolute error (MAE) (see Tayfur and Singh 2006 for definitions), are given in table 2 . The common statistical characteristics (maximum, minimum, average and standard deviation) of the observed and predicted $Q(t)$ values for testing set are given in table 3 . Also the predictions of the proposed LGP and NN models to the observed discharges $(Q(t))$ for testing set are illustrated in figures 2 and 3 , respectively.

Referring to table 2 , it can be stated that the LGP2 model outperformed the other LGP models with the highest $R^{2}(0.691)$ and the lowest MSE $\left(107.914 \mathrm{~m}^{6} / \mathrm{s}^{2}\right)$ and MAE $(0.072)$ values. Another important finding from table 2 is that, LGP and NN models gave the best testing results for input set $\{Q(t-1), Q(t-2)\}$, among corresponding four scenarios given in equations (4)-(7). No significant 
Table 2. Testing results of LGP and NN models.

\begin{tabular}{lccccc}
\hline Model & $\begin{array}{c}\text { MSE } \\
\left(\mathrm{m}^{6} / \mathrm{s}^{2}\right)\end{array}$ & MAE & $R^{2}$ & AIC & $\begin{array}{c}\text { Optimal } \\
\text { architecture }\end{array}$ \\
\hline LGP1 & 127.214 & 4.441 & 0.616 & 1792.74 & - \\
LGP2 & $\mathbf{1 0 7 . 9 1 4}$ & $\mathbf{3 . 9 6 0}$ & $\mathbf{0 . 6 9 1}$ & $\mathbf{1 7 2 8 . 6 9}$ & - \\
LGP3 & 126.374 & 5.170 & 0.615 & 1782.33 & - \\
LGP4 & 140.065 & 6.706 & 0.584 & 1790.33 & - \\
MLPNN1 & 272.35 & 5.168 & 0.615 & 2077.13 & $1-9-1$ \\
MLPNN2 & $\mathbf{2 2 2 . 1 1}$ & $\mathbf{4 . 5 0 9}$ & $\mathbf{0 . 6 7 7}$ & $\mathbf{2 0 5 4 . 1 6}$ & $2-12-1$ \\
MLPNN3 & 295.809 & 7.128 & 0.591 & 2062.03 & $3-7-1$ \\
MLPNN4 & 1970.50 & 8.321 & 0.246 & 3012.91 & $4-23-1$ \\
GRNN1 & 403.095 & 13.206 & 0.419 & 2217.70 & $1-5-1$ \\
GRNN2 & $\mathbf{2 6 2 . 4 8}$ & $\mathbf{6 . 5 6 5}$ & $\mathbf{0 . 6 4 5}$ & $\mathbf{2 1 3 6 . 4 6}$ & $2-9-1$ \\
GRNN3 & 335.067 & 8.235 & 0.517 & 2238.23 & $3-13-1$ \\
GRNN4 & 309.045 & 6.700 & 0.539 & 2296.72 & $4-17-1$ \\
AR(3) & 278.75 & 5.413 & 0.630 & 2067.96 & - \\
\hline
\end{tabular}

discrepancy was observed among the testing results of MLPNN and GRNN models.

Table 3 shows that all of the models failed to predict $Q_{\max }$ in the testing period $\left(305.75 \mathrm{~m}^{3} / \mathrm{s}\right)$, and prediction of LGP2 model is the closest one $\left(141.81 \mathrm{~m}^{3} / \mathrm{s}\right)$. Also, moderate differences were observed among statistical measures of the observed testing set and those of the predicted ones. The underlying reason for this can be explained by the considerable discrepancy observed between statistical characteristics of the training and testing periods.

Referring to the overall performance of all models given in tables 2 and 3, LGP2 can be said to outperform all other models. The simplified analytic form of LGP2 model is given in equation (8), for the purpose of re-evaluation in further studies:

$$
Q(t)=\sqrt{\mathbf{F}_{1}} \times Q(t-1) \cdot 2^{\mathbf{F}_{2}}-3 \mathbf{F}_{2},
$$

where

$$
\begin{aligned}
\mathbf{F}_{1}= & \left(2\left(\mathbf{F}_{3}^{2}-1\right)^{2}-\mathbf{F}_{2}\right) \frac{Q(t-1)}{Q(t-2)}-\frac{Q(t-1)}{Q(t-2)} \\
\mathbf{F}_{2}= & \frac{Q(t-1) Q(t-2)}{-2 Q^{2}(t-2)-4.01 Q(t-2)+2.394}, \\
\mathbf{F}_{3}= & \left(\left(2\left(-\frac{Q(t-1)}{F_{2}}\right)^{0.5}-5.56\right)\right. \\
& \left.\times \frac{\mathbf{F}_{2}}{Q(t-2)}\right)-0.587 .
\end{aligned}
$$

In order to get more reliable evaluation and comparison, $\operatorname{AR}(p)$ model is also used as a traditional method for time-series modelling of daily flow rate. Therefore, the same training and testing datasets utilized in LGP and NN modelling are also employed in developing different $\mathrm{AR}(\mathrm{p})$ models. The AIC results of $\mathrm{AR}(\mathrm{p})$ models, $\mathrm{p}$ ranging from 1 to 10 , for the testing set are illustrated in figure 4. The aim is to select the optimal model, which gives the minimum AIC. It is clearly seen that $\mathrm{AR}(3)$ model, containing $Q(t-1), Q(t-2)$ and $Q(t-3)$ as inputs and $Q(t)$ as output, can be chosen as the optimal AR model with minimum AIC of 2067.96. The testing results of $\mathrm{AR}(3)$ are given in tables 2 and 3 . The results clarify that, the testing results of $\mathrm{AR}(3)$ model can said to be competitive with those of NN models.

\section{Further application}

\subsection{Estimation of flood and minimum discharges}

During the testing period, a number of floods occurred, the maximum of which was recorded on November 17, 2006 with a magnitude of $305.75 \mathrm{~m}^{3} / \mathrm{s}$. The LGP2 model predicted it as $147.81 \mathrm{~m}^{3} / \mathrm{s}$, which is the closest value among those of the proposed models. MLPNN2 model predicted the flood as $143.33 \mathrm{~m}^{3} / \mathrm{s}$ with a second best performance. Table 4 shows the observed and estimated flood and minimum flow values that occurred at testing period. It is clearly seen that, LGP models generally predicted the maximum and minimum discharge values better than the NN models. AR(3) model predictions are observed to be almost as 
Table 3. Statistical performance of the proposed models for testing set.

\begin{tabular}{lrcccc}
\hline & $\begin{array}{c}Q_{\max } \\
\left(\mathrm{m}^{3} / \mathrm{s}\right)\end{array}$ & $\begin{array}{c}Q_{\min } \\
\left(\mathrm{m}^{3} / \mathrm{s}\right)\end{array}$ & $\begin{array}{c}Q_{\text {mean }} \\
\left(\mathrm{m}^{3} / \mathrm{s}\right)\end{array}$ & $\begin{array}{c}S_{x} \\
\left(\mathrm{~m}^{3} / \mathrm{s}\right)\end{array}$ & $C_{v}$ \\
\hline Observed & & & & & \\
$Q(t)\left(\mathrm{m}^{3} / \mathrm{s}\right)$ & 305.75 & 3.20 & 21.67 & 25.69 & 1.19 \\
LGP1 & 83.21 & 3.54 & 20.35 & 21.19 & 1.04 \\
LGP2 & 141.81 & 4.93 & 21.32 & 20.03 & 0.94 \\
LGP3 & 78.92 & 4.23 & 21.79 & 20.45 & 0.94 \\
LGP4 & 83.92 & 6.79 & 23.38 & 21.37 & 0.91 \\
MLPNN1 & 82.08 & 3.61 & 19.86 & 18.07 & 0.91 \\
MLPNN2 & 123.32 & 2.99 & 24.41 & 24.99 & 1.02 \\
MLPNN3 & 82.50 & 4.88 & 21.64 & 18.95 & 0.88 \\
MLPNN4 & 83.87 & 3.88 & 24.24 & 51.12 & 2.11 \\
GRNN1 & 68.14 & 8.28 & 24.19 & 18.71 & 0.69 \\
GRNN2 & 84.76 & 5.84 & 22.50 & 22.57 & 1.00 \\
GRNN3 & 69.34 & 9.32 & 25.81 & 18.33 & 0.71 \\
GRNN4 & 69.84 & 4.36 & 22.02 & 21.12 & 0.96 \\
AR(3) & 112.71 & 6.83 & 22.58 & 30.31 & 1.34 \\
\hline
\end{tabular}

Note: $Q_{\text {mean }}$ - mean observed discharge, $S_{x}$ - standard deviation, $Q_{\min }-$ minimum observed discharge, $Q_{\max }$ - maximum observed discharge, $C_{v}$ - coefficient of variation.
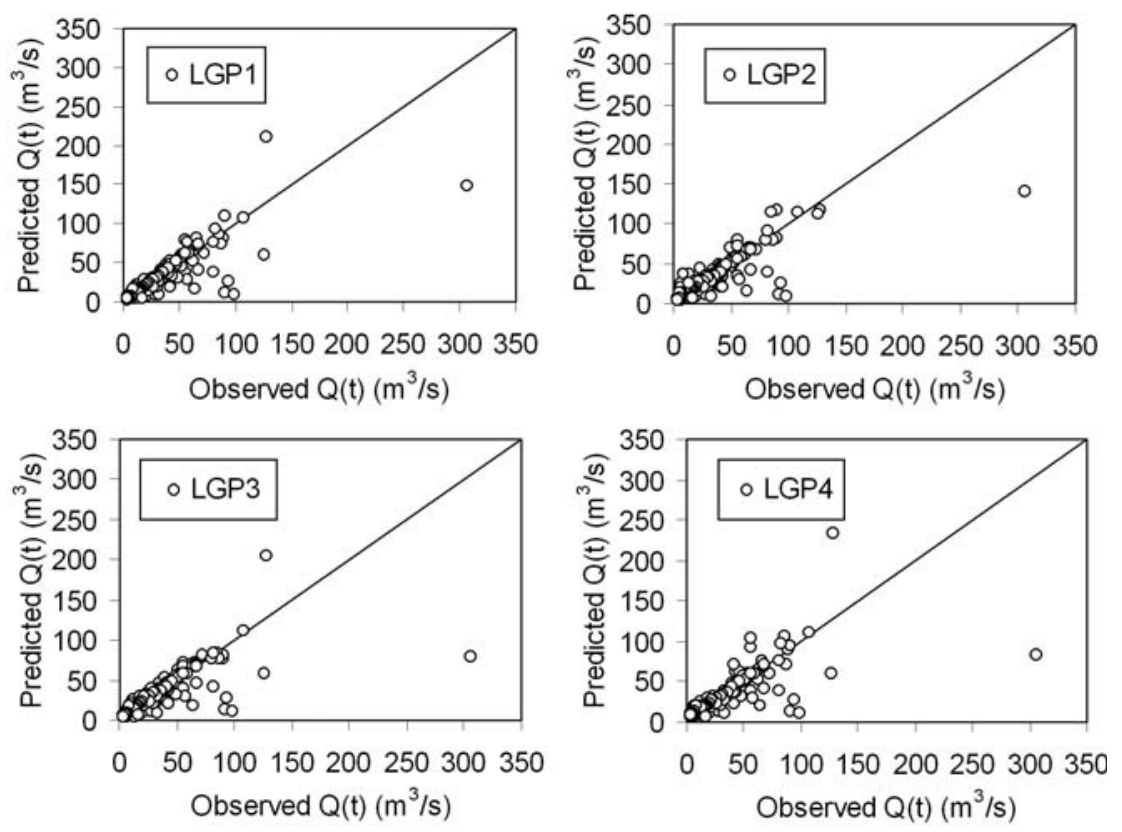

Figure 2. LGP predictions to observed $Q(t)\left(\mathrm{m}^{3} / \mathrm{s}\right)$ values for testing set.

good as LGP2 model in the prediction of maximum $Q(t)$ values, especially better than those for the last five peak discharges, but $\mathrm{AR}(3)$ predictions are much worse than those of LGP2 for minimum $Q(t)$ values (see table 4). LGP models' predictions are closer to observed minimum discharges, compared to other models. As a general view from table 4, the predictions are higher than almost all the observed minimum $Q(t)$ values, whereas all models underpredicted the observed peak $Q(t)$.

\subsection{Generalization of $L G P$ and $N N$ models}

The optimal NN architecture, which is related to the number of neurons in the hidden layer, is one of the most important tasks in MLPNN studies. Generally, the trial and error approach is used. In this study, the best architecture of the network was obtained by a genetic algorithm, which avoids local optimum problems associated with multilayer perceptron modelling, and grant for a global optimal 

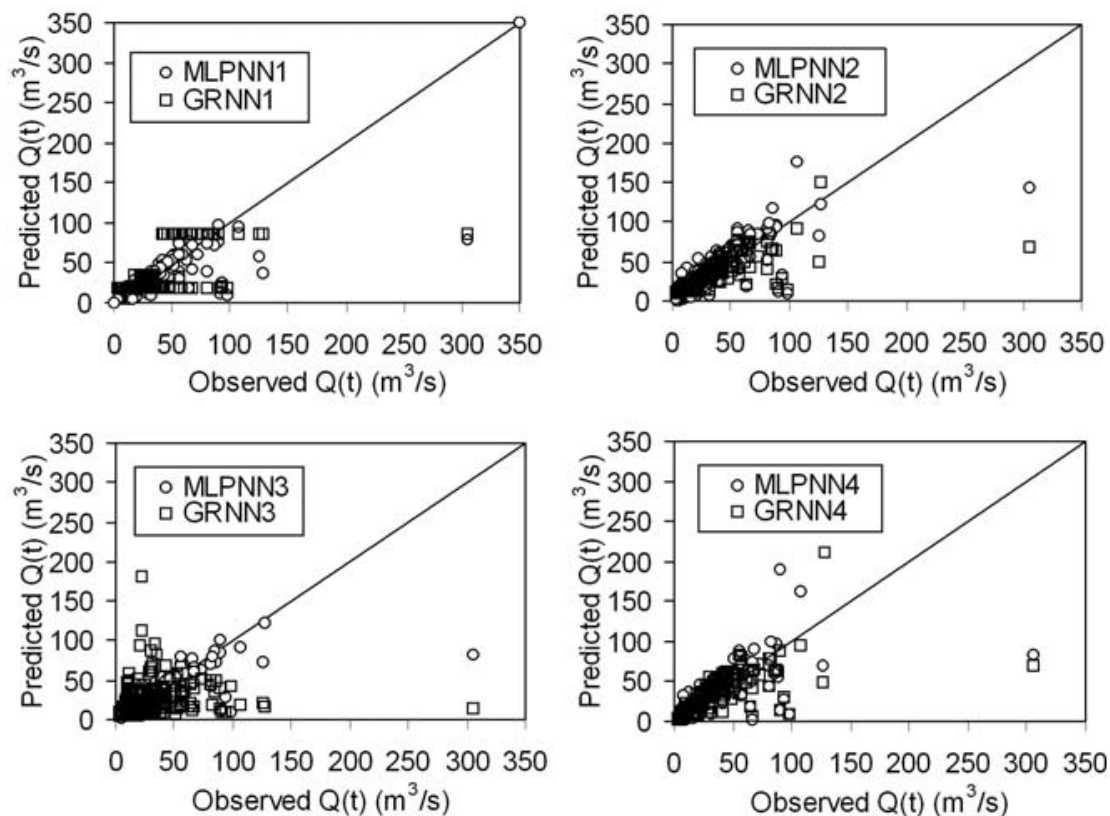

Figure 3. MLPNN and GRNN predictions to observed $Q(t)\left(\mathrm{m}^{3} / \mathrm{s}\right)$ values for testing set.

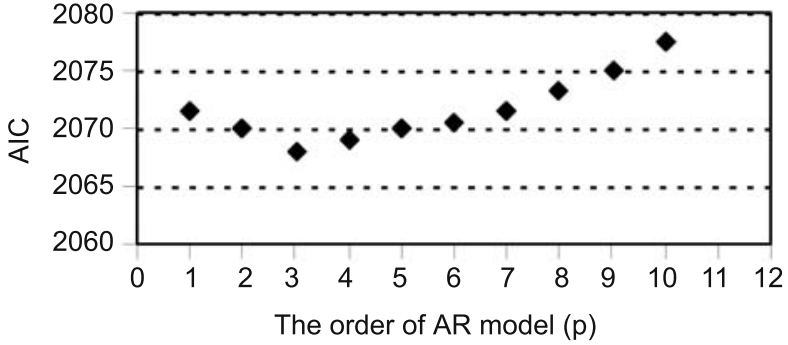

Figure 4. AIC values for $\mathrm{AR}(\mathrm{p})$ models for different orders.

solution. There are several performance criteria for measuring the generalization capacity of soft computing techniques. In this study, Akaike Information Criterion (AIC) defined by Akaike (1974) is utilized to evaluate the robustness of the proposed LGP and NN models.

$$
\mathrm{AIC}=N \ln (\mathrm{MSE})+2 k,
$$

where $N$ is the number of samples in the testing set, and $k$ is the number of network weights.

AIC is used to measure the exchange between testing performance and network size. The goal is to minimize AIC to obtain a network with the best generalization. The AIC values for the predictions of proposed models for testing set is given in table 2. Table 2 also shows the optimal architecture (number of inputs-number of hidden neurons-number of output) of each NN models.

Equation (14) implies that AIC increases with increasing number network weights (i.e., number of hidden neurons in NN models), however, table 2 shows that MLPNN2 has the lowest AIC value as 2054.16 among MLPNN models despite its higher number of neurons (12) than MLPNN1 (7 neurons) and MLPNN3 (9 neurons). This may seem as a contradiction but actually, the lowest MSE $\left(222.11 \mathrm{~m}^{6} / \mathrm{s}^{2}\right)$ of MLPNN2, among other NN models, revealed the lowest AIC $(=2054.16)$ despite the higher number of neurons it employed. Table 5 gives the number of network weights of NN models, which support the above findings.

As seen in table 2, AIC value of LGP2 model is the lowest one among the others, and MLPNN2 model with 2-4-1 architecture, has the lowest AIC value (2054.16) among the NN models.

\subsection{Application}

In this section, the robustness of the proposed LGP and NN models are evaluated based on the application period. In other words, the models proposed in this study are run for another dataset. Time-series of discharge for October 1, 2007 to September 30, 2008 has been taken into consideration for application period. It is emphasized that the data of this period was neither used in the training nor testing period. The statistical results of $Q(t)$ predictions to application data are given in table 5 . LGP2 model has the highest $R^{2}$ as 0.59 , and the lowest MSE as $214.87 \mathrm{~m}^{6} / \mathrm{s}^{2}$. Among the NN models, MLPNN2 can be said to have performed better than the others with $\mathrm{AIC}=2064.63$ and $R^{2}=0.55$ (see table 5).

The observed cumulative hydrograph during the application period and the predictions of 
Table 4. Observed and estimated peak discharge values in testing set.

\begin{tabular}{|c|c|c|c|c|c|}
\hline Date & $\begin{array}{c}Q(t) \\
\left(\mathrm{m}^{3} / \mathrm{s}\right)\end{array}$ & LGP2 & MLPNN2 & GRNN2 & $\operatorname{AR}(3)$ \\
\hline \multicolumn{6}{|c|}{ Maximum discharge values $\left(\mathrm{m}^{3}\right)$} \\
\hline 11.17 .2006 & 305.75 & 147.81 & 143.33 & 68.14 & 112.71 \\
\hline 11.18 .2006 & 127.68 & 117.46 & 111.06 & 149.75 & 105.70 \\
\hline 04.16 .2007 & 125.98 & 113.29 & 82.13 & 49.63 & 109.46 \\
\hline 04.17 .2007 & 107.30 & 115.61 & 176.25 & 91.53 & 110.73 \\
\hline 03.02 .2007 & 98.24 & 10.33 & 9.23 & 14.52 & 76.36 \\
\hline 11.16 .2006 & 93.14 & 25.06 & 31.85 & 29.23 & 83.55 \\
\hline 10.28 .2006 & 90.88 & 12.65 & 12.24 & 16.77 & 96.46 \\
\hline 11.19 .2006 & 89.74 & 82.34 & 93.67 & 22.01 & 95.57 \\
\hline 10.29 .2006 & 89.18 & 117.58 & 97.08 & 62.71 & 96.15 \\
\hline 03.24 .2007 & 86.63 & 80.12 & 116.67 & 65.06 & 75.65 \\
\hline \multicolumn{6}{|c|}{ Minimum discharge values $\left(\mathrm{m}^{3}\right)$} \\
\hline 08.04 .2007 & 3.20 & 4.93 & 5.21 & 10.28 & 6.83 \\
\hline 08.03 .2007 & 3.23 & 4.61 & 5.40 & 10.49 & 6.99 \\
\hline 08.05 .2007 & 3.31 & 5.00 & 4.91 & 10.24 & 6.76 \\
\hline 09.25 .2007 & 3.31 & 5.02 & 5.07 & 10.37 & 7.26 \\
\hline 09.24 .2007 & 3.34 & 5.29 & 6.39 & 10.48 & 5.53 \\
\hline 08.02 .2007 & 3.37 & 4.66 & 1.65 & 11.27 & 7.45 \\
\hline 08.19 .2007 & 3.43 & 5.11 & 6.15 & 10.46 & 4.91 \\
\hline 08.18 .2007 & 3.45 & 5.53 & 6.68 & 10.56 & 7.57 \\
\hline 09.23 .2007 & 3.51 & 5.37 & 6.49 & 10.51 & 7.37 \\
\hline 08.15 .2007 & 3.54 & 5.25 & 6.30 & 10.56 & 7.81 \\
\hline
\end{tabular}

Table 5. Statistical results of model predicitons in application data.

\begin{tabular}{|c|c|c|c|c|}
\hline Model & $\begin{array}{c}\text { MSE } \\
\left(\mathrm{m}^{6} / \mathrm{s}^{2}\right)\end{array}$ & $R^{2}$ & $\mathrm{AIC}$ & $\begin{array}{c}\text { No. of fitting } \\
\text { parameters } \\
(k)\end{array}$ \\
\hline LGP1 & 258.55 & 0.50 & 2051.61 & 12 \\
\hline LGP2 & 214.87 & 0.59 & 1980.06 & 10 \\
\hline LGP3 & 250.21 & 0.55 & 2039.64 & 12 \\
\hline LGP4 & 273.86 & 0.50 & 2076.61 & 14 \\
\hline MLPNN1 & 319.76 & 0.49 & 2149.17 & 22 \\
\hline MLPNN2 & 228.58 & 0.55 & 2064.63 & 41 \\
\hline MLPNN3 & 253.72 & 0.53 & 2088.72 & 34 \\
\hline MLPNN4 & 479.91 & 0.41 & 2497.36 & 122 \\
\hline GRNN1 & 472.70 & 0.40 & 2275.84 & 14 \\
\hline GRNN2 & 323.23 & 0.50 & 2173.11 & 32 \\
\hline GRNN3 & 413.46 & 0.26 & 2314.97 & 58 \\
\hline GRNN4 & 312.55 & 0.48 & 2300.83 & 102 \\
\hline $\operatorname{AR}(3)$ & 310.07 & 0.55 & 2101.93 & 4 \\
\hline
\end{tabular}

LGP2, MLPNN2, GRNN2 and AR(3) models are illustrated in figure 5 . The figure also shows the percentage of error for the corresponding prediction during the application period. LGP2 model is observed to capture the cumulative hydrograph with almost perfect agreement, with
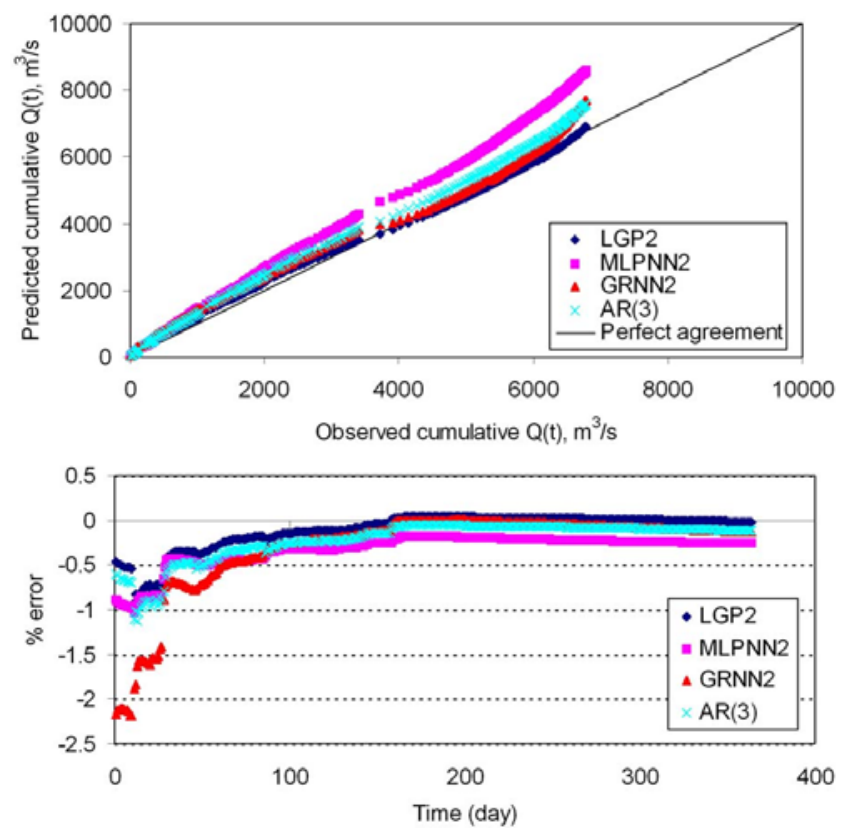

Figure 5. Observed and predicted cumulative hydrograph for application period.

the lowest percentage of error during the whole period. It is clearly seen from the figure that all the models underpredicted the cumulative discharges. LGP2's predictions are clearly seen to be 
closer to the observed ones, compared to other models.

The last column in table 5 shows the number of fitting parameters $(k)$ included in the corresponding model. This column shows that the LGP models have significantly less number of model parameters than the NN models. The proposed LGP models can be attributed as more practical and robust than the NN models, considering the network size and predicting performance together.

\section{Conclusions}

The main goal of this study is to propose LGP as an alternative method of predicting time series of river discharge data, and to evaluate the performance of the proposed LGP models with wellvalidated MLPNN and GRNN techniques. For this purpose, different LGP and NN models have been developed, and the statistical performance of each model was evaluated based on well-known performance measures. The results showed that both LGP and NN techniques predicted the daily time series of discharge with quite good agreement with the observed data. As a conventional method, autoregressive (AR) models were also developed for time-series modelling of the same datasets. As a general view, LGP models performed moderately better than $\mathrm{NNs}$ and $\mathrm{AR}(3)$ models. Especially, in predicting the peak (maximum and minimum) discharge values, LGP2 model was clearly superior to those of NNs and $\mathrm{AR}(3)$. Consequently, the results of this study are very promising and support the use of LGP in predicting the nonlinear and dynamic river flow parameters.

\section{Acknowledgements}

The data used in this study were downloaded from the web server of the USGS. The author wishes to thank the staff of the USGS who are associated with data observation, processing, and management of USGS websites. The financial support of Scientific Research Projects Governing Unit of Gaziantep University during this study is gratefully acknowledged.

\section{References}

Akaike H 1974 A new look at the statistical model identification; IEEE Transactions on Automatic Control 19(6) 716-723.

Aytek A, Guven A, Yuce M İ and Aksoy H 2008 An explicit neural network formulation for evapotranspiration; Hydrol. Sci. 53(4) 893-904.
Aytek A and Alp M 2008 An application of artificial intelligence for rainfall-runoff modelling; J. Earth Syst. Sci. 117(2) 145-155.

Banzhaf W, Nordin P, Keller R and Francone F 1998 Genetic Programming; Morgan Kauffman, San Francisco, CA.

Bhattacharya M, Abraham A and Nath B 2001 A linear genetic programming approach for modelling electricity demand prediction in Victoria; In: Proceedings of the hybrid information systems, first international workshop on hybrid intelligent systems, Adelaide, Australia, 379-393.

Brameier M and Banzhaf W 2001 A comparison of linear genetic programming and neural networks in medical data mining; IEEE Transactions on Evolutionary Computation 5 17-26.

Brameier M 2004 On linear genetic programming; Ph.D. thesis. University of Dortmund.

Baiamonte G and Ferro V 2007 Simple flume for flow measurement in sloping channel; J. Irrig. Drain. Eng. 133(1) 71-78.

Bordignon S and Lisi F 2000 Nonlinear analysis and prediction of river flow time series; Environmetrics $\mathbf{1 1}$ 463-477.

Charhate S B, Deo M C and Sanil Kumar V 2007 Soft and hard computing approaches for real time prediction of currents in a tide dominated area; J. Engineering for the Maritime Environment 221 147-163.

Charhate S B, Deo M C and Londhe S N 2008 Inverse modelling to derive wind parameters from wave measurements; Applied Ocean Research 30(2) 120-129.

Cigizoglu H K and Kisi O 2000 Flow prediction by two back propagation techniques using $\mathrm{k}$-fold partitioning of neural network training data; Nordic Hydrology 36(1) 1-16.

Cigizoglu H K and Alp M 2006 Generalized regression neural network in modelling river sediment yield; Advanced Engineering Software 37 63-68.

Deo O, Jothiprakash V and Deo M C 2008 Genetic Programming to predict spillway scour; Int. J. Tomography and Statistics 8(8) 32-45.

Drecourt J P 1999 Application of Neural Networks and Genetic Programming to Rainfall Runoff Modeling; Danish Hydraulic Institute (Hydro-Informatics Techonologies HIT), D2K-0699-1.

Firat A 2008 Comparison of artificial intelligence techniques for river flow forecasting; Hydrol. Earth Syst. Sci. 12 $123-139$.

Foster J A 2001 Discipulus: A commercial genetic programming system; Genetic Programming and Evolvable Machines 2 201-203.

Gaur S and Deo M C 2008 Real time wave forecasting using genetic programming; Ocean Engineering 35 1166-1172.

Guven A, Gunal M and Cevik A 2006 Prediction of pressure fluctuations on stilling basins; Can. J. Civ. Eng. 33(11) $1379-1388$.

Guven A and Gunal M 2008 A genetic programming approach for prediction of local scour downstream hydraulic structures; J. Irrig. Drain. Eng. 132(4) $241-249$.

Guven A, Aytek A, Yuce M İ and Aksoy H 2008 Genetic programming-based empirical model for daily reference evapotranspiration estimation; CLEAN-Soil, Air, Water 36(10-11) 905-912.

Habib E H and Meselhe E A 2006 Stage-discharge relations for low-gradient tidal streams using data driven models; J. Hydraul. Eng. 132(5) 482-492.

Jain K S 2008 Development of integrated discharge and sediment rating relation using a compound neural network; J. Hydrol. Eng. 13(3) 124-131. 
Jain P and Deo M C 2008 Artificial intelligence tools to forecast ocean waves in real time; The Open Ocean Engineering Journal 1 13-21.

Kalra R and Deo M C 2007 Genetic programming to retrieve missing information in wave records along the west coast of India; Applied Ocean Research 29(3) 99-111.

Kalra R, Deo M C, Kumar R and Agarwal V K 2008 Genetic programming to estimate coastal waves from deep water measurements; Int. J. Ecology and Development 10(8) $67-76$.

Kisi O 2004 River flow modelling using artificial neural networks; J. Hydrol. Eng. 9(1) 60-63.

Kisi O and Cigizoglu H K 2007 Comparison of different ANN techniques in river flow prediction; Civil Engineering and Environmental Systems 24(3) 211-231.

Lopesi H S and Weinert W R 2004 A gene-expression programming system for time series modelling; In: Proc. XXV Iberian Latin American Congress on Computational Methods in Engineering (CILAMCE), Recife, Brazil, CDROM Version.

Maier H R and Dandy G C 2000 Neural networks for the prediction and forecasting of water resources variables: A review of modelling issues and applications; Environ. Modelling Software 15 101-123.

Muttil N and Liong S Y 2004 A superior explorationexploitation balance in shuffled complex evolution; J. Hydraul. Eng. 130(2) 211-220.

Oltean M and Groşan C 2003 A comparison of several linear genetic programming techniques; Complex Systems 14(1) 1-29.

Preis A and Otsfeld A 2008 A coupled model tree-genetic algorithm scheme of flow and water quality predictions in watersheds; J. Hydrol. 349 364-375.

Rumelhart D E, Hinton G E and Williams R J 1986 Learning internal representations by error propagation; In: Paralled Distributed Processing. Explorations in the Microstructure of Cognition (eds) Rumelhart D E, McClelland J L, and the PDP Research Group, Volume 1: Foundations (Cambridge: MIT Press), pp 318-362.
Singh A K, Deo M C and Sanil Kumar V 2007 Neural network - genetic programming for sediment transport; ICE J. Maritime Engineering 160(MA3) 113-119.

Specht D F 1991 A general regression neural network; IEEE Trans. Neural Netw. 2(6) 568-576.

Tayfur G 2002 Artificial neural networks for sheet sediment transport; Hydrol. Sci. 47(6) 879-892.

Tayfur G and Singh V P 2006 ANN and fuzzly logic models for simulating event-based rainfall-ruoff; J. Hydraul. Eng. 132(12) 1321-1330.

Tayfur G and Guldal V 2006 Artificial neural networks for estimating daily total suspended sediment in natural streams; Nordic Hydrology 37 69-79.

Tayfur G, Moramarco T and Singh V P 2007 Predicting and forecasting flow discharge at sites receiving significant lateral inflow; Hydrological Processes 21 $1848-1859$.

Tayfur G and Moramarco T 2008 Predicting hourly-based flow discharge hydrographs from level data using genetic algorithms; J. Hydrol. 352 77-93.

Takens F 1981 Detecting strange attractors in turbulence in turbulence; In: Dynamical systems and turbulence (eds) Hand D and Young L S, Springer-Verlag, Berlin, pp 366.

United States Geographical Survey (USGS) web server: http://www.usgs.gov

Ustoorikar K and Deo M C 2008 Filling up gaps in wave data with genetic programming; Marine Structures 21 177-195.

Wang W, Van Gelder P H A J M and Vrijling J K 2004 Periodic autoregressive models applied to daily streamflow; Proceedings of the 6th International Conference on Hydroinformatics, World Scientific, Singapore, 1334-1341.

Wang W, Van Gelder P H A J M, Vrijling J K and Ma J 2006 Forecasting daily streamflow using hybrid ANN models; J. Hydrol. 324 383-399.

Whigham P A and Crapper P F 2001 Modeling rainfallrunoff using Genetic Programming; Mathematical and Computer Modelling 33 707-721. 